\title{
BMJ Open Perspectives of healthcare providers on the nutritional management of patients on haemodialysis in Australia: an interview study
}

\author{
Jessica Stevenson, ${ }^{1,2}$ Allison Tong, ${ }^{2,3}$ Katrina L Campbell, ${ }^{4}$ Jonathan C Craig, ${ }^{2,3}$ \\ Vincent W Lee ${ }^{1,2,5}$
}

To cite: Stevenson J, Tong A, Campbell KL, et al. Perspectives of healthcare providers on the nutritional management of patients on haemodialysis in Australia: an interview study. BMJ Open 2018;8:e020023. doi:10.1136/ bmjopen-2017-020023

- Prepublication history and additional material for this paper are available online. To view these files, please visit the journal online (http://dx.doi. org/10.1136/bmjopen-2017020023).

Received 10 0ctober 2017 Revised 26 December 2017 Accepted 1 February 2018

Check for updates

${ }^{1}$ Westmead Clinical School, The University of Sydney, Sydney, Australia

${ }^{2}$ Centre for Kidney Research, The Children's Hospital, Westmead, Australia

${ }^{3}$ Sydney School of Public Health, The University of Sydney,

Sydney, Australia

${ }^{4}$ Faculty of Health Sciences and Medicine, Bond University, Robina, Australia

${ }^{5}$ Department of Renal Medicine, Westmead Hospital, Sydney,

Australia

Correspondence to Jessica Stevenson; jste2727@uni.sydney.edu.au

\section{ABSTRACT}

Objective To describe the perspectives of healthcare providers on the nutritional management of patients on haemodialysis, which may inform strategies for improving patient-centred nutritional care.

Design Face-to-face semistructured interviews were conducted until data saturation, and thematic analysis based on principles of grounded theory.

Setting 21 haemodialysis centres across Australia. Participants 42 haemodialysis clinicians (nephrologists and nephrology trainees (15), nurses (12) and dietitians (15)) were purposively sampled to obtain a range of demographic characteristics and clinical experiences. Results Six themes were identified: responding to changing clinical status (individualising strategies to patient needs, prioritising acute events, adapting guidelines), integrating patient circumstances (assimilating life priorities, access and affordability), delineating specialty roles in collaborative structures (shared and cohesive care, pivotal role of dietary expertise, facilitating access to nutritional care, perpetuating conflicting advice and patient confusion, devaluing nutritional specialty), empowerment for behaviour change (enabling comprehension of complexities, building autonomy and ownership, developing self-efficacy through engagement, tailoring self-management strategies), initiating and sustaining motivation (encountering motivational hurdles, empathy for confronting life changes, fostering non-judgemental relationships, emphasising symptomatic and tangible benefits, harnessing support networks), and organisational and staffing barriers (staffing shortfalls, readdressing system inefficiencies).

Conclusions Organisational support with collaborative multidisciplinary teams and individualised patient care were seen as necessary for developing positive patientclinician relationships, delivering consistent nutrition advice, and building and sustaining patient motivation to enable change in dietary behaviour. Improving service delivery and developing and delivering targeted, multifaceted self-management interventions may enhance current nutritional management of patients on haemodialysis.

\section{INTRODUCTION}

Nutritional management for patients on haemodialysis (HD) aims to optimise
Strengths and limitations of this study

- Face-to-face, semistructured interviews were conducted with multidisciplinary clinicians purposively sampled across dialysis units in Australia to obtain indepth and diverse data on their perspectives regarding nutritional management.

- The range of perspectives obtained may inform the development and implementation of future nutrition interventions in haemodialysis.

- Participants were recruited from one country, and therefore transferability to other countries beyond Australia is uncertain.

nutritional status, improve quality of life by minimising symptoms and complications related to excess dietary intake, ${ }^{1}$ and empower patients to manage their dietary needs. Clinicians are tasked to manipulate individual dietary components, including protein, potassium, phosphorus, sodium and fluid, promote general healthy eating, and simultaneously take into consideration comorbid (eg, diabetes, obesity) dietary needs, and patients are expected to adhere to these complex requirements. Non-compliance to dialysis treatment is associated with higher symptom burden, increased medical complications, reduced quality of life and approximately $30 \%$ higher risk of death, ${ }^{2}{ }^{3}$ with non-compliance to diet and fluid restrictions higher $(30 \%-50 \% \text { of patients })^{24}$ than for other elements of treatment. Given the complex and changing nature of nutritional requirements in $\mathrm{HD}$, patients require longterm nutritional counselling using multiple interventions to improve adherence and empower patients to self-manage their dietary needs. ${ }^{5-7}$ Patients want consistent dietary counselling to support their changing dietary needs, however feel that current nutritional counselling is inadequate. ${ }^{89}$ 
Renal multidisciplinary teams, comprising nephrologists, nurses and dietitians, play a pivotal role in providing nutritional counselling; thus, there is a need to understand health professionals' beliefs and attitudes regarding nutritional management within the context of clinical care. However, there is limited literature on renal clinicians' experiences and perceptions regarding nutritional management in patients on HD. Identifying the challenges, gaps and inefficiencies in current practice can inform appropriate changes to service provision and the development of interventions to support dietary change in patients. The aim of this study is to describe renal healthcare providers' perspectives in the nutritional management of patients on HD to guide patient care, service delivery and the design of interventions to improve nutritional and other important patient outcomes.

\section{METHODS}

We followed the Consolidated criteria for Reporting Qualitative research framework to report this study. ${ }^{10}$

\section{Participant selection}

Nephrologists (including nephrology trainees), nurses and dietitians, with experience in providing care for adults on HD in Australia, were eligible to participate. Participants were purposively selected to include a range of age, gender, years of experience in nephrology, practice locations and size of HD units. Participants were initially identified from the investigators' collegial networks, and a snowball technique was also used whereby participants could nominate other clinicians who they believed might offer a unique or important perspective on this topic. The research team contacted potential participants via email who were suggested by colleagues or other participants.

\section{Data collection}

The interview guide was developed to include questions and prompts on clinical decision-making and approaches to nutritional management (online supplementary file 1). Researcher JS conducted semistructured interviews inperson at hospitals, conferences, venues or by telephone, from April 2016 to November 2016 until data saturation was achieved within each discipline (ie, nephrology, nursing and dietetics). All interviews were digitally audio-recorded and transcribed verbatim.

\section{Data analysis}

Using the principles of grounded theory and thematic analysis, JS coded the transcripts using HyperRESEARCH software (ResearchWare V.3.3, USA) and inductively identified concepts relating to decision-making in the nutritional management. Similar concepts were grouped into themes, with patterns, broader overarching concepts and links among the concepts searched for by one author (JS) and checked by another (AT). The research team discussed preliminary findings and then invited participants to comment (ie, member checking). Participant feedback was coded and incorporated into revisions of the analytical framework.

\section{RESULTS}

Of the 51 participants contacted, $42(83 \%)$ participated and included nephrologists $(\mathrm{n}=11,26 \%)$, nephrology trainees $(n=4,10 \%)$, dietitians $(n=15,36 \%)$ and nurses $(\mathrm{n}=12,29 \%)$ from 21 dialysis centres across New South Wales ( $n=33)$, Queensland $(n=7)$ and Victoria $(n=2)$. Reasons for clinicians not participating were due to clinical commitments $(n=4)$ or non-response to invitation $(\mathrm{n}=5)$. On average, the interviews lasted approximately $30 \mathrm{~min}$ and were conducted inperson $(62 \%)$ or on the telephone $(38 \%)$. Participant characteristics are provided in table 1.

We identified six major themes: responding to changing clinical status; integrating patient circumstances; delineating specialty roles in collaborative structures; empowerment for behaviour change; initiating and sustaining motivation; and organisational and staffing barriers. For each theme, the subthemes are described below. The themes relate to participants across all disciplines unless specified. A thematic schema illustrating the relationships between themes is shown in figure 1. Illustrative quotes are provided in table 2 .

\section{Responding to changing clinical status}

Individualising strategies to patient needs

Dietary needs in HD were seen as 'dynamic' and needed 'individualized' recommendations to meet patients' clinical needs. Participants took into account patients' quality of life and survival, as well as clinical indicators such as biochemistry, fluid status, symptomatology and comorbidities, to guide recommendations. Participants noted that while patient education primarily focused on standard topics (dietary electrolytes, fluid and protein), they attempted to avoid a 'one size fits all approach' and 'blanket' recommendations.

\section{Prioritising acute events}

Nephrologists and nurses agreed that 'acute', 'life-threatening' issues, such as vascular access complications, were prioritised above 'preventative' dietary needs. Hyperkalaemia and fluid overload were seen as 'acute' issues needing immediate attention because of the association with cardiovascular disease and mortality. In contrast, 'long term consequences' such as malnutrition, obesity, phosphorous control and dietary quality were seen to be of relatively lower priority.

\section{Adapting guidelines}

Participants reported multiple and conflicting guidelines created uncertainty and confusion when providing nutritional advice. They depended on clinical judgement, past experience and contextualised guidelines based on treatment goals and comorbidities. Some were sceptical about the impact of nutrition, particularly dietary phosphate 


\begin{tabular}{lc}
\hline Table 1 Participant characteristics & \\
\hline Characteristics & $\mathbf{n}(\%)$ \\
\hline Role & \\
\hline Nephrologist & $11(26)$ \\
\hline Nephrology trainees & $4(10)$ \\
\hline Nurse & $12(28)$ \\
\hline Dietitian & $15(36)$ \\
\hline Sex & \\
\hline Male & $9(22)$ \\
\hline Female & $33(78)$ \\
\hline Age (years) & \\
\hline $20-29$ & $7(17)$ \\
\hline $30-39$ & $12(28)$ \\
\hline $40-49$ & $13(31)$ \\
\hline $50-59$ & $8(19)$ \\
\hline $60-69$ & $2(5)$ \\
\hline Experience in haemodialysis (years) & \\
\hline $0-5$ & $13(31)$ \\
\hline $6-10$ & $8(19)$ \\
\hline $11-15$ & $2(5)$ \\
\hline $15+$ & $19(45)$ \\
\hline Size of dialysis unit (number of patients) \\
\hline $1-50$ & $10(24)$ \\
\hline $51-100$ & $5(12)$ \\
\hline $101-200$ & $16(38)$ \\
\hline $201-300$ & $3(7)$ \\
\hline $301-400$ & $1(2)$ \\
\hline $401-500$ & $2(5)$ \\
\hline Go0+ & $5(12)$ \\
\hline Necation of dialysis unit & \\
\hline Rural/regional & \\
\hline
\end{tabular}

restriction on critical outcomes, such as survival and cardiovascular disease, and thus did not emphasise this in their practice. Dietitians felt that guidelines were too restrictive, and did not account for dietary quality or nutrient bioavailability and chose to deviate from guidelines, particularly when patients had conflicting clinical needs.

\section{Integrating patient circumstances}

Assimilating life priorities

Participants reported taking into account patients' values, goals, quality of life, and social and personal circumstances when providing nutrition education. Participants changed the focus of counselling based on treatment goals, with comprehensive nutritional counselling for transplant candidates, while for older patients they focused on symptom management and quality of life. Among dietitians, some were flexible with restrictions to allow patients to enjoy food particularly in consideration of patients' priorities, cultural needs and circumstances.

\section{Access and affordability}

Dietitians and nurses raised concerns about financial insecurity limiting access to appropriate, 'healthy' foods for patients. In rural and remote areas, participants highlighted that the high cost of 'healthy' foods and lack of clean tap water were barriers to changing patients' choices. Some felt helpless in areas where patients did not have the opportunity to choose appropriate nutrition and believed that there were no realistic strategies available to them to overcome these barriers.

\section{Delineating specialty roles in collaborative structures Shared and cohesive care}

Participants regarded nutritional care as 'everyone's business' and required 'co-management' from all of the multidisciplinary team. Collaborative care was felt to be enhanced by face-to-face interactions and regular communication. Participants felt that the role of the nephrologist and nurse was to introduce nutritional issues, provide general education and facilitate access to a dietitian, whereas the dietitian's role was to provide specific, tailored education, particularly to complex patients. While being primarily responsible for patients' nutritional management, dietitians reported being influenced by nephrologists' attitudes to ensure a cohesive approach and 'not butt heads'.

\section{Pivotal role of dietary expertise}

Nutrition was seen as a vital component of treatment and by some to be as important as dialysis and medications. Participants believed that nutritional intake had direct (eg, symptom burden) and indirect (eg, higher pill burden) impacts on patients. Participants viewed dietitians as the experts who could provide detailed, practical advice that was necessary to support dietary changes. Dietitians felt better equipped than other staff to assimilate patients' nutritional needs and priorities and reported using active engagement techniques such as health coaching, individualised dietary plans and shopping guides to build patients' self-management skills.

\section{Perpetuating conflicting advice and patient confusion}

Providing consistent messages to patients was perceived to be important; however, participants reported differing nutritional priorities often led to conflicting advice being given. Some dietitians perceived that nurses and nephrologists communicated restrictive, 'black and white' dietary recommendations to patients, which they speculated was due to inadequate nutrition knowledge. Participants also observed that patients accessed information from the 


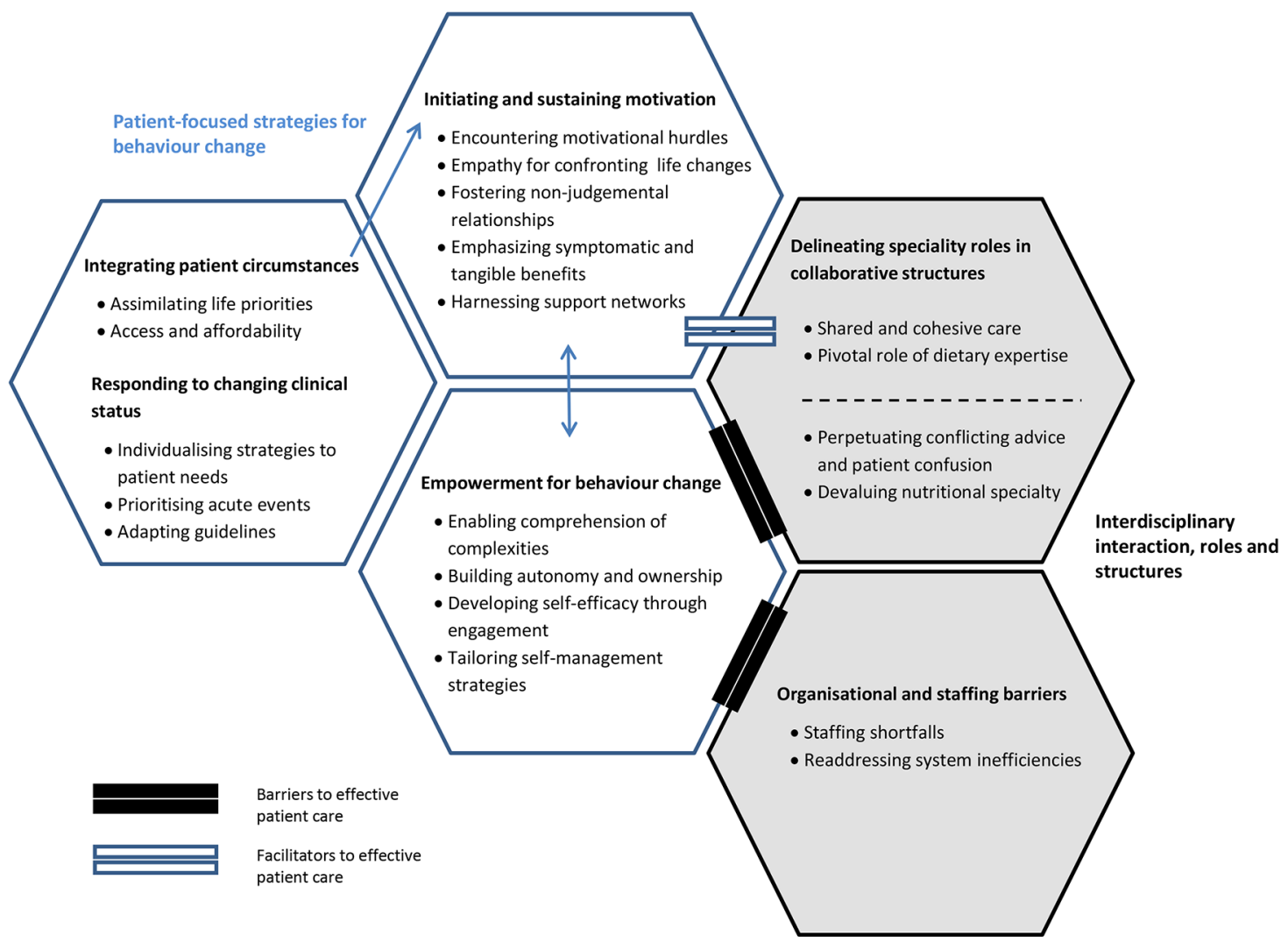

Figure 1 Thematic schema. Participants regarded nutritional management as an important component of care. Individualisation of care through integrating patients' personal, social and cultural circumstances was central to clinicians' decision-making and delivery of care. Supporting patients to take ownership of their disease management, and develop selfefficacy and self-management skills was seen as paramount. However, ineffective multidisciplinary team communication and splintered interteam dynamics, coupled with inadequate staffing and resources, were felt to limit the implementation of necessary behaviour change strategies. Lack of motivation and the ability to sustain motivation were seen as significant challenges, and were attributed to factors such as geographical location, education attainment, financial security and social support. Providing shared care and access to dietary experts were seen to facilitate patients' understanding of the renal diet and helped to develop and maintain motivation.

internet or complementary medicine professionals (eg, naturopaths), which contributed to patients' confusion.

\section{Devaluing nutritional specialty}

Some perceived that nutritional care was often deprioritised and could be seen as an 'after-thought'. Participants reported there was variable support for the role of nutrition, particularly among nephrologists. Some dietitians felt they needed to 'prove' themselves to be knowledgeable or have an established professional relationship before their input was sought. Dietitians generally felt there was a lack of understanding among nephrologists and nurses about their role and scope of practice and were frustrated when their advice seemed to be undermined.

\section{Empowerment for behaviour change \\ Enabling comprehension of complexities}

Participants described the renal diet as 'dynamic' with 'complex scientific concepts', which could be 'over-clinicalized', making it difficult for patients to comprehend. Patients with comorbidities or who had multiple nutritional issues (eg, electrolytes and fluid) were observed to become 'overwhelmed', less motivated and more likely to disengage. Participants believed that nutrition counselling needed to be introduced earlier to allow patients to become familiar with the diet and 'set the tone' for dietary change. Participants discussed the importance of providing positively framed, 'relevant' and 'holistic' nutrition messages to allow patients to adopt changes.

\section{Building autonomy and ownership}

Participants felt that patients have become institutionalized' in the healthcare system and should be encouraged to become active in their healthcare. Strategies and goal setting should be patient-driven to create 'ownership' and accountability. Participants did not see it as their role to ensure adherence to the diet but to support patients to make 'informed choices'.

\section{Developing self-efficacy through engagement}

Providing patients with a 'road-map' for behaviour change by building patients' confidence, self-efficacy and problem-solving skills was seen as paramount. Setting small 
Table 2 Illustrative quotes

Theme Illustrative quotations

Responding to changing clinical status

Individualising strategies "It depends a little bit on the patient and what their main problems are. Whether they are

to patient needs overweight, underweight, malnourished, nourished, active, inactive, have problems with their potassium, diabetic." (Nephrologist)

Prioritising acute events "Acute medical problems are at the forefront, rather than focusing on preventative medicine, which is what I think nutrition is." (Nephrologist)

"You can kill with fluid overload; you can kill with high potassium." (Nephrologist)

Adapting guidelines "It's finding that balance between what is clinically appropriate and best dietetically with what is appropriate for a patient." (Dietitian)

Integrating patient circumstances

Assimilating life priorities "It is the whole picture. It is diet, it is sleep, it is their sex life, it is putting needles in their arms, and it is the 12-year-old daughter. You do have to take all of that in." (Nurse)

"Helping them find realistic choices, despite the fact that they may be higher in phosphorus, in the big picture they're going to be better off eating than not eating." (Dietitian)

Access and affordability "What hope do you have in trying to make healthy dietary changes when it easier to buy white bread and a couple of bucks worth of chips, versus fruit and vegetables that are almost going off and are frightfully expensive." (Dietitian)

"It is really difficult to make appropriate food choices when you have got nothing there." (Dietitian)

Delineating specialty roles in collaborative structures

Shared and cohesive "Making sure that I'm keeping my own integrity in terms of what I know is important and what I care prioritise, but going in line with what the nephrologist thinks as well. I think that helps us work as a team more, rather than be butting heads." (Dietitian)

"I will work with what I know are acceptable limits. So if one nephrologist is very liberal with something, like potassium for instance, in his patients I am not going to be (restrictive). It does influence my practice." (Dietitian)

Pivotal role of dietary "Ultimately if you don't put it (food) in in the first place you don't have to rectify it on dialysis or expertise with pills." (Nephrologist)

"I think where the dietitian is adding to the team is looking what they are currently eating, how can we modify and what options do they have." (Nephrologist)

Perpetuating conflicting "It's the challenge of multiple arms of advice that a patient needs to try comply with. And that can advice and patient confusion change from even doctor to doctor, some person may emphasize more, the next person may not, so the patient thinks it's ok we can have whatever we want." (Nephrologist)

"I would have nurses telling patients one thing, doctors telling them the other thing, and me telling them the third thing." (Dietitian)

Devaluing nutritional "It's interesting some of the things that doctors say to you, you think really? You don't realize that specialty that's part of the role we play?" (Dietitian)

"I feel like it is often the last thing on their (nephrologists') list. Given that patients also have multiple co-morbidities that they are juggling and they are referred to so many other health practitioners, I think allied health get left out." (Dietitian)

"I have worked with several dietitians that I think have made a difference, but it has not been consistent. One problem is that we have very junior dietitians." (Nephrologist)

"Anyone will be aware of the importance that diet plays in renal disease, but I can't say if all nephrologists appreciate the value the dietitian brings." (Nephrologist)

Empowerment for behaviour change

Enabling comprehension "If they can't understand the importance of all the different nutritional factors then that makes of complexities it really hard for them. I mean why would you be motivated if you can't really understand it?" (Dietitian)

"I have found my approach to educating the patients about the importance of these (nutrition) things would arrive too late. So they've already ended up in hospital because they haven't stuck to their fluid restriction and then it's educating them." (Nephrologist)

"If you are talking out of their realm of understanding, not comprehension, but it's not relevant to their lives, it is mute advice, it doesn't help." (Nephrologist)

"I think the only way you get people to change their behaviour is by selling them on the message, why there is a benefit to changing. People won't do things that are difficult for no reason." (Nephrologist) 
Table 2 Continued

\begin{tabular}{|c|c|}
\hline Theme & Illustrative quotations \\
\hline $\begin{array}{l}\text { Building autonomy and } \\
\text { ownership }\end{array}$ & $\begin{array}{l}\text { "These are adults, they make their own decisions. We provide opportunity, we provide education." } \\
\text { (Nephrologist) } \\
\text { "I think if a patient makes a choice and the choice is informed in the context of their situation, then } \\
\text { l'm happy to go along with it." (Nephrologist) }\end{array}$ \\
\hline $\begin{array}{l}\text { Developing self-efficacy } \\
\text { through engagement }\end{array}$ & $\begin{array}{l}\text { "Have them set their own goals with our guidance, so that we can achieve that step by step." } \\
\text { (Dietitian) } \\
\text { "It's often working on small swaps, so trying to be very practical and realistic in the things that you } \\
\text { are going to encourage them to have." (Dietitian) } \\
\text { "I try and target just one thing that is the main issue. I think we all overload our patients with } \\
\text { information and they don't absorb it." (Nephrologist) } \\
\text { "Praising small goals, setting small goals for people and rewarding people with praise. So that } \\
\text { positive reinforcement." (Nurse) }\end{array}$ \\
\hline $\begin{array}{l}\text { Tailoring self- } \\
\text { management strategies }\end{array}$ & $\begin{array}{l}\text { "Giving them clear instructions and clear guidance and options." (Nephrologist) } \\
\text { "I think some strategies are more effective on certain patients than others. A patient who is } \\
\text { struggling, then compromise may be a better strategy. Patients who appear to not have any } \\
\text { interest that's when my strategy will be explaining the outcomes. It's trial and error." (Nephrologist) } \\
\text { "You're } 5 \text { L over, pick up } 5 \mathrm{~kg} \text { of oranges, carry them around for a few hours and then imagine } \\
\text { that's what your heart is doing." (Nurse) }\end{array}$ \\
\hline
\end{tabular}

Initiating and sustaining motivation

Encountering "I find it incredibly frustrating and it doesn't feel like a good use of time, and it's not fulfilling as a motivational hurdles dietitian to keep banging your head against a wall." (Dietitian)

"Trying to get them to see the need and the importance behind the dietary changes, coz they feel well or they don't understand how the diet can impact on how they feel." (Dietitian)

Empathy for confronting "They've got other things going on that are important and that's valid really isn't it? I can see that life changes nutrition isn't always my highest value thing in my life either, so I can empathize." (Dietitian) "There is a mum who has lots of children who frequently misses dialysis. I have tried the carrot with her, the stick with her but at the end of the day she is a full mother, her husband is in and out of jail, and she has to be there for her kids. I can't change that." (Nephrologist)

Fostering non- "When you're first meeting a patient, it's not about diet; it's about establishing a relationship." judgemental (Dietitian)

relationships "Sometimes nutrition is not going to be the patient's priority, but always leaving that door open so that they know that you haven't just dismissed them." (Dietitian)

"You don't want to get to the point where you get that push back and you lose the rapport and they just see you as the fun police." (Dietitian)

Emphasising symptomatic and tangible benefits

"One lady was telling me her legs were just so heavy she found it hard to walk around. So I linked that education with that she was fluid overloaded all of the time." (Dietitian)

"You only need 1 or 2 times to be in hyperkalaemia in CCU to realize it is clearly life-threatening so they change the way (they eat)." (Nephrologist)

Harnessing support "The patient might understand and then they go home and mum is cooking or the wife's cooking networks and if she doesn't also have an understanding of this then obviously that's going to be critical to their outcome as well." (Nurse)

"I try and link the family in with this diet as well. So having limited salt is not just for you because you are a renal patient, it's good for your whole family." (Dietitian)

Organisational and staffing barriers

Staffing shortfalls "We are then not getting to go through and do a general follow up or anything like that; it feels a little bit like band-aiding." (Dietitian)

"I actually had tears a couple of times because I just feel like I can't assess my patients properly." (Nurse)

"Since I have come into this position I feel like I am drowning." (Dietitian)

Readdressing system "Our traditional approach is not working, so we need to change." (Nephrologist)

inefficiencies

"If their (eGFR) 15 you are too late, it (nutrition education) needs to start even before pre-dialysis, in primary health care." (Nurse)

"The primary deficit is we haven't communicated with them (patients) in the first place." (Nephrologist) 
realistic goals, encouraging self-monitoring, providing positive feedback and health coaching were strategies employed by participants. Given the complexity of the diet, conducting regular reviews and giving actionable advice were felt to be important to enhance patients' motivation and self-efficacy.

\section{Tailoring self-management strategies}

Participants reported 'no one strategy fits all' and some reported using a variety of approaches to help improve patients' self-management. Passive (eg, behaviour and consequence education) and active (eg, interactive resources and feedback, motivational interviewing) strategies were used depending on the 'root of the problem'. Participants tried to match strategies with patients' stage of change, health literacy, cognition and cultural needs. Future suggestions for interventions to improve patient engagement included using reminder systems and visual cues (eg, posters in dialysis units), developing educational DVDs and cookbooks, enhancing the nursing role in nutrition education, and using technology such as mobile phone apps and online forums. Barriers to developing and implementing new strategies included lack of time, meeting the needs of culturally diverse populations and age appropriateness of technology-based interventions.

\section{Initiating and sustaining motivation \\ Encountering motivational hurdles}

Participants discussed their struggle with patients' lack of motivation or 'will-power' to make dietary changes. This lack of motivation left participants feeling 'frustrated' and like 'banging your head against a wall'. Sometimes clinicians perceived this lack of motivation as patients 'giving up' once they reached dialysis or that patients felt they had 'gotten away with it'. Participants highlighted patients needed to be self-driven and internally motivated to make long-term dietary changes.

\section{Empathy for confronting life changes}

Participants were empathetic to the difficulty of sustaining long-term motivation. Complex dietary needs, the overwhelming nature of dialysis and personal circumstances were perceived to impact on patients' ability to sustain motivation and maintain self-care. Participants noted change in patients' self-identity, lifestyle, financial circumstances and social roles (eg, as parents or carers) impacted on their ability to engage in their healthcare. Participants saw that deviations from recommendations were 'human nature', and it was important to balance dialysis treatment restrictions, including nutrition, with other factors in patients' lives.

\section{Fostering non-judgemental relationships}

Developing long-term, non-judgemental relationships with patients and families was seen as crucial to gaining patients' trust and changing behaviour. Participants focused on developing rapport and personal relationships with patients and emphasised the importance of showing broader interest in their life, and allowing disengaged patients to reconnect to services. Participants felt patients sought advice from clinicians who gave the most time and attention, are familiar to them and those who were culturally similar.

\section{Emphasising symptomatic and tangible benefits}

Participants encouraged behaviour change through linking perceptible symptoms or benefits and physical impacts to how patients felt to help improve motivation and give context to dietary recommendations. Motivating patients to change dietary behaviours associated with acute symptoms or consequences (eg, fluid overload and hyperkalaemia) was seen to be easier.

\section{Harnessing support networks}

Participants found that involving family and other significant support people, particularly the primary cook, in dietary education better enabled patients to change their eating behaviours. Dietitians reported trying to 'normalize' dietary restrictions that could be adopted by the family. Social isolation was seen to be associated with poorer dietary behaviours and choices.

\section{Organisational and staffing barriers \\ Staffing shortfalls}

Nursing and dietetic participants were frustrated with inadequate staffing and resources, with some feeling overwhelmed in their workloads. Inadequate staffing resulted in suboptimal, disjointed care, with inadequate time to follow up patient care plans or to provide health coaching. Some dietitians felt there was inadequate time for professional development or to participate in quality improvement or research activities that could improve their practice or service delivery. Nurses reported to have become 'task-driven' and felt inadequate staffing leads to neglect of holistic care.

\section{Readdressing system inefficiencies}

Participants felt that nutritional care needed to be remodelled and clinicians needed to 'work smarter'. Nurses and nephrologists wanted access to experienced dietitians who comprehended the complexities of the renal diet, and some felt dietetic services could be refocused to educate patients 'before pre-dialysis' in 'primary care'. Improving multidisciplinary team collaboration and patient-clinician communication was also seen as paramount by all disciplines.

\section{DISCUSSION}

Renal clinicians regarded nutritional management as an important component of care in HD that required individualised strategies through integrating personal, social and cultural circumstances of patients. They emphasised the need to support patients in developing self-efficacy and self-management skills to become autonomous and take ownership of their dietary management. However, ineffective multidisciplinary team communication and splintered interteam dynamics, coupled with inadequate 
staffing and resources, were barriers to implementing behaviour change strategies and also perpetuated conflicting advice and patient confusion. They also found it challenging when patients were perceived to lack motivation to make dietary changes, which they attributed to difficulties in comprehension of complex concepts, poor access to healthy food (in low-socioeconomic and remote communities), low education attainment, financial constraints and the absence of social support. Shared care and access to dietary experts were seen to facilitate patients' understanding and acceptance of the renal diet and helped to develop and maintain motivation.

Differences in the approach and attitudes to nutrition management were apparent across disciplines, with nephrologists and nurses reporting that they addressed nutritional issues in an ad-hoc manner and reactively based on biochemistry and symptomatology because of time constraints and prioritising acute clinical needs. In contrast, dietitians aimed to provide regular support using individualised, active behaviour change strategies (eg, food exchanges, health coaching) to pre-empt, identify and manage nutritional issues early. Dietitians believed that regular, gradual counselling was necessary to avoid overwhelming patients with too much information and helped enable patients to adopt dietary changes. However, dietitians felt they were under-resourced with inadequate time to effectively educate and support patients.

In our study, clinicians felt that conflicting advice given to patients diminished their understanding of the diet and created ambivalence towards the impact and role of diet. Differing nutritional priorities and disjointed nutritional advice were reported to result from confusion due to the multiplicity of conflicting guidelines and resources, inconclusive nutrition research and out-of-date literature.

Clinicians, particularly dietitians, working in rural and remote areas felt that patient care and their ability to affect clinical outcomes was impaired due to service limitations from working across large geographical areas, patients' overt financial insecurity and lack of access to appropriate foods. These challenges have also been reflected by experiences of service providers working with Aboriginal patients on HD living in geographically isolated areas, where lack of appropriate public health

Table 3 Recommendations for clinical practice

\begin{tabular}{|c|c|}
\hline Domain & Suggested strategies and actions \\
\hline Organisational environment & $\begin{array}{l}\text { Enhance team dynamics } \\
\text { Encourage development of unique roles and responsibilities }{ }^{20} \text { for medical, nursing and dietetic } \\
\text { disciplines } \\
\text { - Encourage professional development and training to build expertise }{ }^{27} 28 \\
\text { roles } \\
\text { - Train and support dietitians to reach proficient and expert levels of practice }{ }^{27} \\
\text { - Undertaking postgraduate renal nutrition training } \\
\text { - Mentoring with senior dietitians } \\
\text { - Training with nephrologists and nurses to develop non-nutrition, clinical knowledge } \\
\text { Facilitate team building through regular communication and shared work spaces in or near } \\
\text { dialysis units } \\
\text { Service delivery } \\
\text { Facilitate continuity of care of dietetic services through extended rotation schedules or } \\
\text { permanent roles within clinical specialties } \\
\text { Support research } \\
\text { Prioritisation of shared research and quality improvement activities into core business of } \\
\text { multidisciplinary team }\end{array}$ \\
\hline Self-management support & $\begin{array}{l}\text { Patient engagement } \\
\text { Engage with patients and communities to determine needs to develop appropriate } \\
\text { interventions } \\
\text { - Enhance patient understanding and ownership } \\
\text { - Provide nutrition counselling before commencement of haemodialysis } \\
\text { - Simplify nutrition messages }{ }^{914} \\
\text { - Provide practical advice that is culturally, socially and personally relevant }{ }^{911} 14 \\
\text { Develop a toolkit of self-management strategies to implement multifaceted interventions that } \\
\text { can be individualised to patient needs }{ }^{5928} \\
\text { Develop dialysis unit education materials (eg, educational posters, newsletters, topical poster } \\
\text { boards) } \\
\text { Explore technology-based interventions to support and enhance current nutrition education } \\
\text { in haemodialysis }\end{array}$ \\
\hline
\end{tabular}


initiatives, logistical issues (eg, lack of transportation) and inadequate staffing and resources limited patient care. ${ }^{11}$

Clinicians reported being significantly challenged and frustrated by patients' perceived lack of motivation and resistance to dietary change. Clinicians working in peritoneal dialysis ${ }^{12}$ and kidney transplantation ${ }^{13}$ have similarly reported they believe that poor dietary and lifestyle habits hinder effective treatment, ${ }^{12}{ }^{13}$ while intrinsic motivation and support from social networks enabled behaviour change. ${ }^{12}$ Developing motivation and engaging patients and their families are particularly important in HD as diet and fluid regimens are more restrictive than for other treatments. Renal clinicians in our study felt that comprehensive counselling with an experienced dietitian was an important element of care and helped to motivate patients to make appropriate dietary changes. Likewise, studies exploring patients' experience have reported that patients prefer dietary education from experts, such as renal dietitians, who can provide the necessary practical advice and constructive feedback. ${ }^{914}$ Equally, establishing mutually respectful and trusting relationships helps patients to become empowered and engaged in their disease management, ${ }^{11} 1315$ and interventions and counselling that develop knowledge, build self-efficacy and provide regular monitoring have been associated with greater treatment adherence in HD patients. ${ }^{16} 17$

The complexity of the renal diet demands that clinicians have the knowledge and skills to provide flexible, culturally and personally relevant nutrition counselling. The importance of providing simplified, personalised counselling that normalises dietary recommendations within the family and social environments was emphasised in our study and has been reflected by studies exploring patient $^{91415}$ and clinician ${ }^{11}$ experience. Interventions to enhance self-management should be individualised, patient-driven and focused on skill development and sustaining motivation. Structured, multifaceted educational interventions that incorporate elements such as behavioural contracts, feedback based on self-monitoring, portion size awareness and clinician follow-up are seen to enhance patient self-management and outcomes. ${ }^{56} 18$ Self-management programmes are best conducted within a collaborative multidisciplinary team environment ${ }^{19}$ with positive interteam communication and improving continuity of care and patients' experience. ${ }^{11}{ }^{20}$ However, in our study inadequate staffing and resources led to clinicians feeling underequipped to provide the necessary, complex counselling, and in some instances undermining of dietary advice and perceived underappreciation of the dietitians' role led to fractured team dynamics and inconsistent nutrition messages being disseminated.

The development and adoption of time and resource efficient behaviour change interventions to support clinicians and patients is needed. Technology-based interventions have been gaining increasing attention and provide a new opportunity to engage with people to improve health behaviours, including those in geographically isolated and low socioeconomic areas. There is limited literature reporting the use of technology-based interventions to change dietary behaviours in chronic kidney disease. However, systematic reviews exploring the impact of technology-based interventions have reported improved dietary behaviours in coronary heart disease $(\mathrm{CHD})^{21}$ and similar or improved outcomes in a range of $\mathrm{CHD}^{22} 23$ and diabetes ${ }^{24-26}$ clinical outcomes when using mobile phone, web and telemedicine interventions.

Our study documents a wide spectrum of opinions and experiences of a diverse group of renal clinicians, which enabled comparisons across disciplines; however, there are some potential limitations. Our participants were all English-speaking and were based in Australian dialysis units, which may limit transferability of our results. However, similarities with these findings and studies conducted in other countries such as the USA and UK suggest that our findings may be broadly applicable. ${ }^{1320}$ However, the relevance of some of the concepts in our study to other regions with different cultural and social norms is uncertain.

Providing united and shared care, building positive and collaborative multidisciplinary team relationships, and fostering positive patient-clinician relationships are pivotal aspects to be addressed. Changes to service delivery and adopting new behaviour change interventions to effectively support patients in managing their disease need to be considered. Based on our findings and previous literature, table 3 outlines our suggestions and recommendations for change in HD dietary management.

There is a need for high-quality research to investigate the efficacy of self-management interventions. Telehealth, mobile phone, computer or internet interventions are gaining rapid and widespread traction and offer clinicians new means of patient interaction, which may be cost and time efficient, and reduce barriers such as time constraints and geographical location. Systematic reviews have been limited to low-quality evidence, and there is little guidance for practice. ${ }^{23-26}$ Gaining an understanding of patients' perspectives regarding the renal diet, and perceived barriers and strategies that help improve self-efficacy and self-management is needed to ensure interventions meet patients' needs.

Renal healthcare providers view dietary management as a critical component of patient care; however, suboptimal multidisciplinary team relationships and organisational barriers result in disjointed care, contribute to patient confusion and ambivalence, and limit the implementation of self-management strategies. Supporting patients to take ownership of their disease and being able to effectively manage their dietary needs was seen by clinicians as essential to improving care on HD. Adoption of new service delivery models and self-management interventions is needed to enhance the nutritional management of patients on HD.

Acknowledgements We thank all participants for sharing their interesting thoughts and perspectives for this study. 
Contributors JS participated in the design of the study, conducted the interviews, transcribed the interviews, carried out thematic analysis and drafted the manuscript. AT, KLC, JCC, VWL designed the study, participated in the thematic analysis and provided critical review of the manuscript. All authors made substantial contributions to conception and design, acquisition of data, or analysis and interpretation of data; drafting the article or revising it critically for important intellectual content; and provided final approval of the version to be published. All authors had full access to all of the data in the study and can take responsibility for the integrity of the data and the accuracy of the data analysis.

Funding JS is supported by a National Health and Medical Research Council Better Evidence and Translation in Chronic Kidney Disease (BEAT-CKD) Program Grant (1092579).

Competing interests None declared.

Patient consent Not required.

Ethics approval All participants provided written and voluntary informed consent. The study was approved by the University of Sydney Ethics Committee (20130616).

Provenance and peer review Not commissioned; externally peer reviewed.

Data sharing statement № additional data are available.

Open Access This is an Open Access article distributed in accordance with the Creative Commons Attribution Non Commercial (CC BY-NC 4.0) license, which permits others to distribute, remix, adapt, build upon this work non-commercially, and license their derivative works on different terms, provided the original work is properly cited and the use is non-commercial. See: http://creativecommons.org/ licenses/by-nc/4.0/

(C) Article author(s) (or their employer(s) unless otherwise stated in the text of the article) 2018. All rights reserved. No commercial use is permitted unless otherwise expressly granted.

\section{REFERENCES}

1. Campbell K, Rossi M, Carrero JJ. Nutrition Management in Hemodialysis. In: Magee CC, Tucker JK, Singh AK, eds. Core concepts in dialysis and continuous therapies. Boston, MA: Springer US, 2016:53-70.

2. Baines LS, Jindal RM. Non-compliance in patients receiving haemodialysis: an in-depth review. Nephron 2000;85:1-7.

3. Durose CL, Holdsworth M, Watson V, et al. Knowledge of dietary restrictions and the medical consequences of noncompliance by patients on hemodialysis are not predictive of dietary compliance. $J$ Am Diet Assoc 2004;104:35-41.

4. Kim Y, Evangelista LS. Relationship between illness perceptions, treatment adherence, and clinical outcomes in patients on maintenance hemodialysis. Nephrol Nurs J 2010;37:271-81.

5. Lopez-Vargas PA, Tong A, Howell M, et al. Educational interventions for patients with CKD: a systematic review. Am J Kidney Dis 2016;68:353-70.

6. Desroches S, Lapointe A, Ratté S, et al. Interventions to enhance adherence to dietary advice for preventing and managing chronic diseases in adults. Cochrane Database Syst Rev 2013;2:Cd008722.

7. Fouque $D$. Why is the diet intervention so critical during chronic kidney disease? J Ren Nutr 2003;13:173.

8. Hollingdale R, Sutton D, Hart K. Facilitating dietary change in renal disease: investigating patients' perspectives. J Ren Care 2008;34:136-42

9. Meuleman Y, Ten Brinke L, Kwakernaak AJ, et al. Perceived barriers and support strategies for reducing sodium intake in patients with chronic kidney disease: a qualitative study. Int J Behav Med 2015;22:530-9.
10. Tong A, Sainsbury P, Craig J. Consolidated criteria for reporting qualitative research (COREQ): a 32-item checklist for interviews and focus groups. Int J Qual Health Care 2007;19:349-57.

11. Rix EF, Barclay L, Wilson S, et al. Service providers' perspectives, attitudes and beliefs on health services delivery for Aboriginal people receiving haemodialysis in rural Australia: a qualitative study. BMJ Open 2013;3:e003581.

12. McCarthy A, Cook PS, Fairweather C, et al. Compliance in peritoneal dialysis: a qualitative study of renal nurses. Int $J$ Nurs Pract 2009;15:219-26.

13. Spigner C, Lyles CR, Galvin G, et al. A qualitative assessment of personal and social responsibility for kidney disease: the increasing kidney disease awareness network transplant project. J Natl Med Assoc 2011;103:879-84.

14 de Brito-Ashurst I, Perry L, Sanders TA, et al. Barriers and facilitators of dietary sodium restriction amongst Bangladeshi chronic kidney disease patients. J Hum Nutr Diet 2011;24:86-95.

15. Palmer SC, Hanson CS, Craig JC, et al. Dietary and fluid restrictions in CKD: a thematic synthesis of patient views from qualitative studies. Am J Kidney Dis 2015;65:559-73.

16. Oka M, Chaboyer W. Influence of self-efficacy and other factors on dietary behaviours in Japanese haemodialysis patients. Int J Nurs Pract 2001;7:431-9.

17. Oh HS, Park JS, Seo WS. Psychosocial influencers and mediators of treatment adherence in haemodialysis patients. J Adv Nurs 2013;69:2041-53.

18. Mason J, Khunti K, Stone M, et al. Educational interventions in kidney disease care: a systematic review of randomized trials. Am J Kidney Dis 2008;51:933-51.

19. Bonner A, Havas K, Douglas C, et al. Self-management programmes in stages 1-4 chronic kidney disease: a literature review. J Ren Care 2014;40:194-204.

20. Swallow V, Smith T, Webb NJA, et al. Distributed expertise: qualitative study of a British network of multidisciplinary teams supporting parents of children with chronic kidney disease: Distributed expertise in a network of multidisciplinary teams. Child Care Health Dev 2015;41:67-75.

21. Kelly JT, Reidlinger DP, Hoffmann TC, et al. Telehealth methods to deliver dietary interventions in adults with chronic disease: a systematic review and meta-analysis. Am J Clin Nutr 2016;104:1693-702.

22. Chow CK, Redfern J, Hillis GS, et al. Effect of lifestyle-focused text messaging on risk factor modification in patients with coronary heart disease. JAMA 2015;314:1255.

23. Widmer RJ, Collins NM, Collins CS, et al. Digital health interventions for the prevention of cardiovascular disease: a systematic review and meta-analysis. Mayo Clin Proc 2015;90:469-80.

24. Zhai YK, Zhu WJ, Cai YL, et al. Clinical- and cost-effectiveness of telemedicine in type 2 diabetes mellitus: a systematic review and meta-analysis. Medicine 2014;93:e312.

25. Pal K, Eastwood S, Michie S. Computer based diabetes selfmanagement interventions for adults with type two diabetes mellitus. Cochrane Database Syst Rev 2013;28.

26. Cotter AP, Durant N, Agne AA, et al. Internet interventions to support lifestyle modification for diabetes management: A systematic review of the evidence. J Diabetes Complications 2014;28:243-51.

27. Kent PS, McCarthy MP, Burrowes JD, et al. Academy of Nutrition and Dietetics and National Kidney Foundation: revised 2014 standards of practice and standards of professional performance for registered dietitian nutritionists (competent, proficient, and expert) in nephrology nutrition. J Acad Nutr Diet 2014;114:1448-57.

28. Desroches S, Lapointe A, Deschênes SM, et al. Dietitians' perspectives on interventions to enhance adherence to dietary advice for chronic diseases in adults. Can J Diet Pract Res 2015;76:103-8. 